\title{
The Immediate Effects of Single Leg Bridge Exercise on Abdominal Muscle Activity in Subacute Stroke Patients : a Preliminary Study
}

\author{
Hoyoung Kim ${ }^{a}{ }^{\oplus}$, Chanbum Park ${ }^{b}$, Sooyong Bang ${ }^{b}(\infty$, \\ Hoyoung Jang ${ }^{\mathrm{c}} \odot$, Yongju Kim ${ }^{\mathrm{d}} \oplus$, Sukmin Lee ${ }^{\circledR}$ \\ aDepartment of Physical Therapy, The Graduate School, Sahmyook University, Seoul, Republic of Korea \\ ${ }^{b}$ Department of Physical Therapy, Kyunghee University Medical Center, Seoul, Republic of Korea \\ 'Department of Physical Therapy, Catholic University Eunpyeong St. Mary's Hospital, Seoul, Republic of Korea \\ d The Graduate School of Integrated Medicine, CHA Medical University, Pocheon, Republic of Korea \\ eDepartment of Physical Therapy, College of Health and Welfare, Sahmyook University, Seoul, Republic of Korea
}

\begin{abstract}
Objective: Single leg bridge exercise (SLBE) improves trunk muscle activation and provides muscle stability by aligning joints related to posture. This preliminary study aimed to investigate the effects of SLBE on abdominal muscle activation in subacute stroke patients.
\end{abstract}

Design: Cross-sectional study.

Methods: Fifteen subacute stroke patients ( 9 males, 6 females) voluntarily participated in this study. SLBE was performed on the affected side. Each individual patient performed an SLBE ten times in three sets and was asked to hold their position for $5 \mathrm{~s}$. Muscles activity was recorded using a surface electromyography (EMG) system before and after the SLBE. A surface EMG system was used to analyze the muscle activity during general bridge exercise, including the rectus abdominis (RA), internal oblique (IO), and external oblique (EO) abdominal muscles.

Results: Muscle activity of both EO and IO on the affected side significantly increased $(\mathrm{p}<0.05)$, whereas the muscle activity of the RA and the three muscles on the unaffected side did not show a significant difference. In addition, improvement in muscle asymmetry of the EO and IO showed a significant change after SLBE $(\mathrm{p}<0.05)$.

Conclusions: SLBE is effective in activating the abdominal muscles of stroke patients on the affected side and is a helpful exercise intervention that activates the muscles to transform asymmetric abdominal muscles into symmetric patterns.

Key Words: Electromyography, Exercise, Abdominal muscles, Muscle activation, Stroke

\section{Introduction}

Stroke is one of the leading causes of death in adults and a major cause of movement disorders due to damage to the cerebral blood vessels caused by ischemia or hemorrhage [1]. Neurological and functional recovery occurs rapidly over 6 months during the subacute phase [2]. Trunk control ability is an important predictive factor for functional recovery in stroke patients; therefore, engaging in active rehabilitation to improve trunk control ability is important for the functional recovery in the early stage of stroke rehabilitation [3].

The trunk muscles in stroke patients, like other limbs, demonstrate an abnormal pattern in which muscle activation of the unaffected and affected sides

Received: May 12, 2021 Revised: Jun 6, 2021 Accepted: Jun 14, 2021

Corresponding author: Sukmin Lee (ORCID https://orcid.org/0000-0002-6062-956X)

Department of Physical Therapy, College of Health and Welfare, Sahmyook University

815, Hwarang-ro, Nowon-gu, Seoul, 01795 Republic of Korea [01795]

Tel: + 82-10-3704-5650 Fax: + 82-2-3399-1639 E-mail: leesm@syu.ac.kr

This is an Open-Access article distributed under the terms of the Creative Commons Attribution Non-Commercial License (http://creativecommons.org/licenses/ by-nc/4.0) which permits unrestricted non-commercial use, distribution, and reproduction in any medium, provided the original work is properly cited.

Copyright @ 2021 Korean Academy of Physical Therapy Rehabilitation Science 
demonstrates imbalance [4]. The loss of trunk control ability is a factor that not only undermines optimal body posture and extremity functioning but also impairs activities of daily living including balance and gait abilities [5].

Because trunk muscles are controlled by both cerebral hemispheres, brain lesions on one side can affect abdominal muscles on both sides, and a low level of muscle activation on the affected side causes decreased muscle function [6].

The muscles that stabilize and support the trunk are the multifidus, transverse abdominis, and internal oblique (IO), classified as deep stabilizer muscles, and the erector spinae, rectus abdominis (RA), and external oblique (EO), classified as superficial stabilizer muscles [7]. Co-activation between the deep stabilizer muscles and superficial stabilizer muscles is required to maintain trunk muscle stability [7].

Trunk stabilization exercises improve normal gait patterns, help regain feelings on the lower extremity of the affected side, and improve symmetry of the pelvic alignment, which facilitates normal motor function and reduces excessive muscle tone in stroke patients [5].

Bridge exercise is a trunk stabilization exercise known to improve motor control and co-activation of the trunk muscles. It has been conducted using diverse methods and modifications to enhance effectiveness [8-17].

According to previous studies $[12,13]$, the rotational torque of the torso during single leg bridge exercise (SLBE) improves trunk muscle activation and provides muscle stability by aligning joints related to posture. Abdominal muscles are more important in improving the rotational torque than the back muscles of the lumbar-thoracic region [14].

Bridge exercise also contributes to improving balance and gait in stroke patients [15], and SLBE is associated with improvement in static balance in stroke patients [16]. SLBE has been widely applied in rehabilitation treatments, but there are insufficient studies to confirm the effect of SLBE on abdominal muscle activation in patient with stroke, and no studies have applied on the affected side or only to patients with subacute phase.

Therefore, this study is to determine the effects of SLBE on the activation of abdominal muscle in subacute stroke patients.

\section{Method}

\section{Subjects}

Fifteen participants who were admitted to $\mathrm{M}$ Rehabilitation Center in Gyeonggi-do Province participated in the exercise program.

Subjects were selected according to the following criteria: (1) patients with stroke within 6 months of onset, (2) patients who could understand and follow simple verbal instructions and scored over 24 scores in the Mini-Mental State Examination-Korean version, (3) patients who could independently perform the bridge exercise, and (4) patients who could perform the curl-up posture enough to lift the scapula off the floor.

The exclusion criteria were: (1) patients with unilateral neglect, (2) patients with impaired proprioception, (3) patients with other musculoskeletal and neurological disorders, apart from stroke. The characteristics of the subjects are as follows table (Table 1).

Table 1. Characteristics of subjects $(n=15)$

\begin{tabular}{lll}
\hline Characteristic & & Subjects \\
\hline Sex & Male & $9(60.0)$ \\
& Female & $6(40.0)$ \\
Age (years) & & $59.87(3.77)$ \\
Height (cm) & & $163.60(7.01)$ \\
Weight (kg) & & $63.47(8.35)$ \\
Onset (month) & & $3.47(0.74)$ \\
Affected side & Right & $6(40.0)$ \\
& Left & $9(60.0)$ \\
Lesion location & ACA & $1(6.7)$ \\
& MCA & $8(53.3)$ \\
& PCA & $3(20.0)$ \\
& Pons & $2(13.3)$ \\
& Thalamus & $1(6.7)$ \\
Lesion type & Infarction & $12(80.0)$ \\
& Hemorrhage & $3(20.0)$ \\
MMES-K (score) & & $26.33(0.617)$ \\
\hline
\end{tabular}

Values are presented as $\mathrm{n}(\%)$ or mean (SD)

ACA: Anterior cerebral artery, MCA: Middle cerebral artery,

PCA: Posterior cerebral artery, MMES-K: Mini-Mental State Examination-Korean version 
All subjects explained the procedure and purpose of the study and received consent forms when the subject was voluntarily signed. This study was approved by institutional review board in the Bioethics Committee of Sahmyook University (2-1040781-A-N-012020054HR).

\section{Measurement}

\section{EMG data collection}

Muscle activity was measured using a surface electromyography (EMG) system (Figure 1). The sampling rate of the EMG signal was set to $2,000 \mathrm{~Hz}$, and the band-pass filtering was set at $20-450 \mathrm{~Hz}$ [18]. To reduce skin resistance to the EMG signal, hair was removed from the skin on the attachment site, the stratum corneum of the skin was eliminated by rubbing it three to four times with sandpaper, and the skin was cleaned with ethanol alcohol.

Electrodes were located on both side of the RA, EO, and IO [18]. (1) To measure RA activity, electrodes were placed on the muscle belly midway between the pubis and the umbilicus; (2) to measure EO activity, electrodes were located $5 \mathrm{~cm}$ above the anterior superior iliac spine (ASIS); and (3) to measure IO activity, electrodes were placed $2 \mathrm{~cm}$ medial to the ASIS (Figure 2).

\section{Measuring the reference voluntary contraction (RVC) value}

To measure the reference voluntary contraction (RVC) value for each patient, all participants
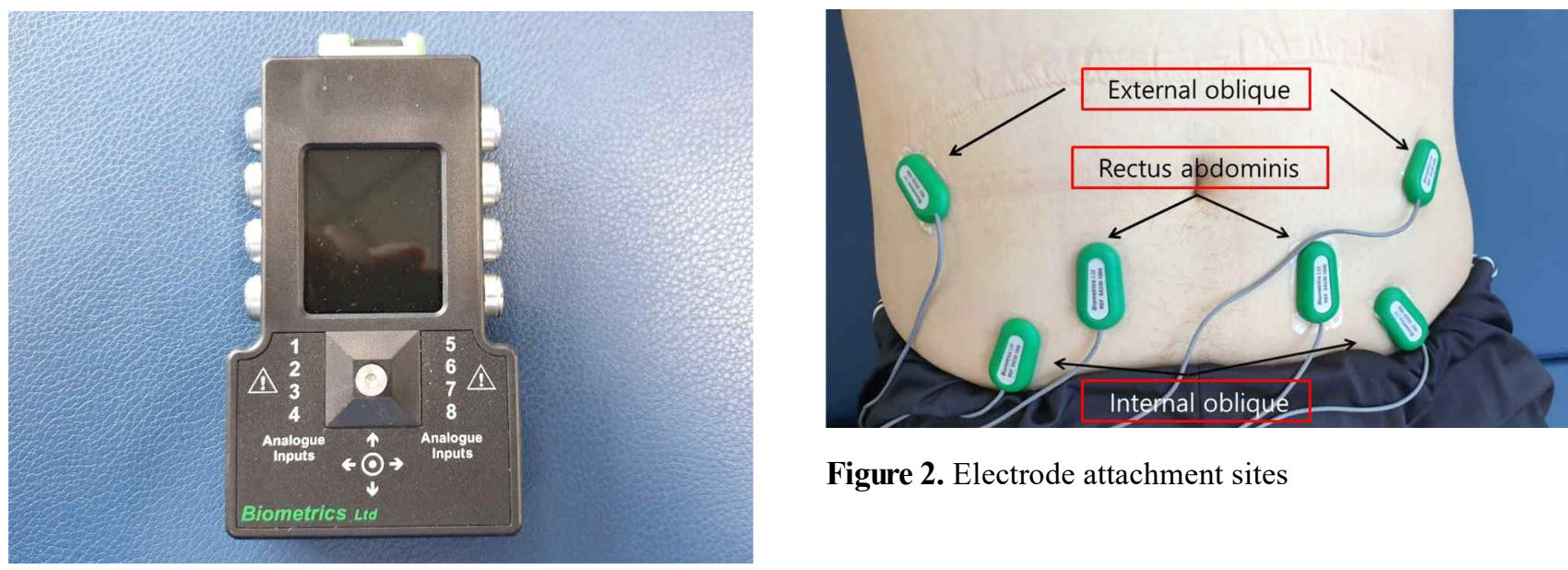

Figure 2. Electrode attachment sites performed three types of curl-up positions [19]. The participants laid down on the mat with their feet on the floor to maintain a $45^{\circ}$ hip joint flexion and a $90^{\circ}$ knee flexion. (1) To record the EMG data of the RA, a curl-up position was maintained for $5 \mathrm{~s}$ with both scapula lifted off the floor. (2) To record the right side of the EO and left side of the IO, a curl-up position in which the right scapula was lifted off the floor was maintained for 5 s. (3) To record the left EO and right IO, the curl-up position was maintained for $5 \mathrm{~s}$ with the left scapula lifted off the floor. The average value of the 3-s position where the patient was asked to hold his/her pose, excluding the values at the first and last second, measured three times for each posture, was determined as the RVC value.

\section{Procedures}

All subjects underwent the general bridge exercise (GBE) before and after SLBE. Muscle activation of the RA, EO, and IO was measured using the surface EMG system during each GBE before and after SLBE. The percentage of RVC (\%RVC) for each muscle before and after the exercise intervention was calculated by comparing the measured value to that of the RVC value. Muscle asymmetry was assessed by inputting raw data in the following formula: (unaffected side - affected side) / (unaffected side + affected side $) \times 100$. Muscles are more symmetric as the values near zero.

Figure 1. Surface EMG (DataLog MWX8, Biometrics Ltd., UK) 


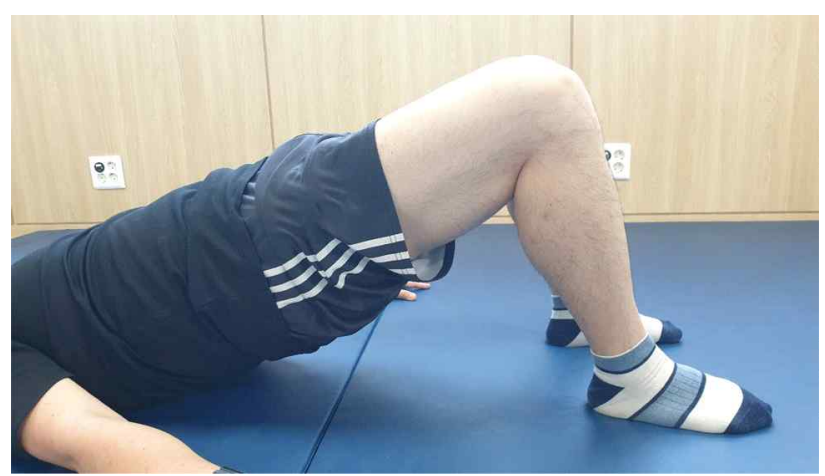

Figure 3. General bridge exercise

\section{General bridge exercise (GBE)}

In the lying position, the patients placed both arms on their sides, shoulder width apart at $30^{\circ}$. Maintaining a $45^{\circ}$ hip joint flexion and a $90^{\circ}$ knee flexion, the hips were raised as much as possible until the body was straight so that the hip joint angle was at $0^{\circ}$ (Figure 3). This posture was maintained for $5 \mathrm{~s}$. The values measured during $3 \mathrm{~s}$ of the $5 \mathrm{~s}$ were used, excluding the values for the first and last second [18]. The average value of EMG data was obtained by calculating the mean of the values of during the $3 \mathrm{~s}$ described above, measured three times while the patient repeated the GBE posture described above [20].

\section{Single leg bridge exercise (SLBE)}

In the lying position, the patients put both arms on their sides, shoulder width apart at $30^{\circ}$. The leg on the affected side was pressed on the floor, maintaining a $45^{\circ}$ hip joint flexion and $90^{\circ}$ knee flexion, while the leg on the unaffected side is fully stretched out and laid comfortably down on the floor. To avoid compensation, subjects received verbal instructions on performing the SLBE as follows: "Raise up your affected side hip. and stay calm your both arms and unaffected side leg." Then the hips were raised as much as possible until the body was straight so that the hip joint angle was at $0^{\circ}$ (Figure 4). This posture was maintained for $5 \mathrm{~s}$ and repeated ten times in three sets. The resting time was $3 \mathrm{~min}$ between each set.

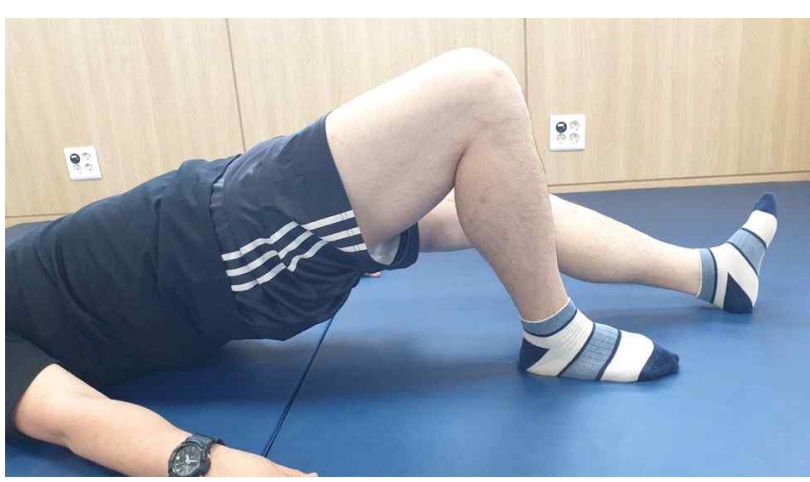

Figure 4. Single-leg bridge exercise

\section{Data and statistical analysis}

The SPSS (ver 21.0, IBM Co., USA) was used to analyze the EMG data for this study. The normality test was verified using the Shapiro-wilk test. To analyze the difference before and after the exercise intervention, a paired t-test was performed. Statistical significance was set at $\mathrm{p}<0.05$.

\section{Result}

\section{Differences in muscle activities pre- and postexercise}

Abdominal muscle activation before and after the exercise intervention was compared, and improvement in the muscle activation of the EO and IO on the affected side was statistically significant $(p<0.05)$. The RA on the affected side and the three muscles on the unaffected side showed no significant changes (Table 2, Figure 5).

\section{Differences in muscle asymmetry pre- and postexercise}

Muscle asymmetry before and after the exercise intervention was assessed; the muscle asymmetry of both the EO and IO was significantly improved ( $p$ $<0.05)$. RA demonstrated no statistically significant changes (Table 3, Figure 6). 

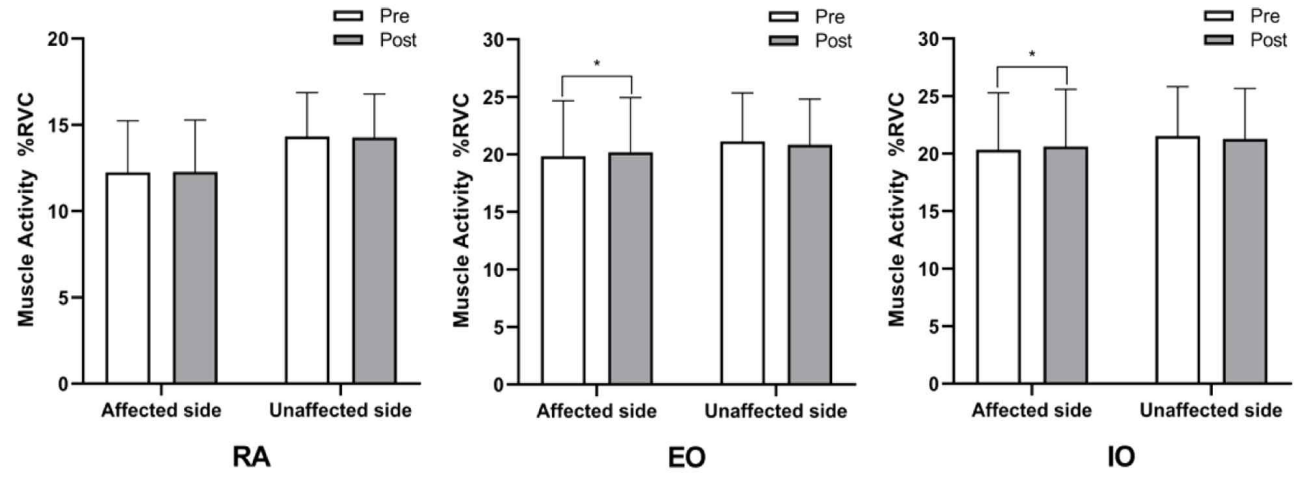

Figure 5. Differences in muscle activities pre- and postexercise.

RVC: reference voluntary contraction, RA: rectus abdominis, EO: external oblique, IO: internal oblique. $* \mathrm{p}<0.05$

Table 2. Differences in muscle activities pre- and postexercise

\begin{tabular}{llllll}
\hline \multirow{2}{*}{ Muscle } & & \multicolumn{2}{l}{ Mean \%RVC(SD) } & \multirow{2}{*}{ t-value } & \multirow{2}{*}{ p-value } \\
\cline { 2 - 4 } Are & Post & & \\
\hline \multirow{2}{*}{ Affected side } & RA & $12.25(2.98)$ & $12.28(3.00)$ & -0.893 & 0.387 \\
& EO & $19.85(4.81)$ & $20.19(4.74)$ & -2.479 & $0.027^{*}$ \\
& IO & $20.34(4.95)$ & $20.61(4.99)$ & -2.191 & $0.046^{*}$ \\
\hline \multirow{2}{*}{ Unaffected side } & RA & $14.33(2.53)$ & $14.27(2.52)$ & 1.174 & 0.260 \\
& EO & $21.14(4.20)$ & $20.85(3.97)$ & 1.544 & 0.145 \\
& IO & $21.53(4.29)$ & $21.29(4.38)$ & 1.401 & 0.183 \\
\hline
\end{tabular}

Values are presented as mean (SD)

RVC: reference voluntary, RA: rectus abdominis, EO: external oblique, IO: internal oblique $* \mathrm{p}<0.05$

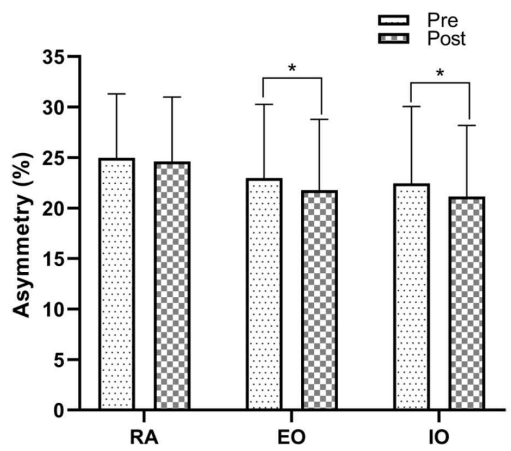

Figure 6. Differences in muscle asymmetry pre- and postexercise. RA: rectus abdominis, EO: external oblique, IO: internal oblique. $* p<0.05$

Table 3. Differences in muscle asymmetry pre- and postexercise

\begin{tabular}{|c|c|c|c|c|}
\hline \multirow{2}{*}{ Muscle } & \multicolumn{2}{|c|}{ Mean \%unit'(SD $\left.{ }^{\mathrm{b}}\right)$} & \multirow{2}{*}{ t-value } & \multirow{2}{*}{ p-value } \\
\hline & Pre & Post & & \\
\hline $\mathrm{RA}^{\mathrm{c}}$ & $24.98(6.32)$ & $24.62(6.37)$ & 2.079 & 0.056 \\
\hline $\mathrm{EO}^{\mathrm{d}}$ & $22.97(7.30)$ & $21.78(6.70)$ & 2.481 & $0.026^{*}$ \\
\hline $\mathrm{IO}^{\mathrm{e}}$ & $22.46(7.60)$ & $21.15(7.03)$ & 3.128 & $0.007^{*}$ \\
\hline
\end{tabular}

a (unaffected side - affected side) / (unaffected side + affected side) $\times 100$

Values are presented as mean ( ${ }^{b}$ standard deviation), ${ }^{c}$ rectus abdominis, ${ }^{\mathrm{d}}$ external oblique, ${ }^{\mathrm{e}}$ internal oblique $* \mathrm{p}<0.05$ 


\section{Discussion}

This is a preliminary study for a prospective study comparing the muscle activation of the RA, EO, and IO on both sides of the body before and after the exercise intervention using surface EMG to examine the immediate effects of SLBE on abdominal muscle activation in subacute stroke patients.

Bridge exercise is an easy and simple exercise that can be performed without other instruments. Therefore, it is widely used in clinical treatments as an effective exercise intervention method to retain muscle co-activation [20]. SLBE is more effective than GBE because trunk muscle activation is further induced to compensate for only one of the two supporting legs being used [21-22].

Patients who have suffered a stroke experience a reduction in trunk control abilities due to preceding low muscle activation in the trunk and decreased muscle strength [23]. The patients perform activities of daily living with a higher dependence level on the unaffected side rather than the affected side, which weakens the muscles on the affected side because it is used less [24]. This contributes to muscle asymmetry, resulting in muscle weakening [23-24]. Therefore, the ideal goal of rehabilitation in stroke patients is to reduce the asymmetry of muscles on each side of the body [25].

According to previous studies, 4 weeks of SLBE in which the affected leg was raised improved weight-bearing and balance abilities during standing in stroke patients [16]. In a cross-sectional study conducted in healthy adults, muscle activation of the RA, EO, IO, and erector spinae on the side of the leg was significantly improved, and the activation levels were greatest in the erector spinae [11].

In addition, many studies have compared the effects of various bridge exercises in adults; muscle activation of the IO on the elevated side and the erector spinae on both sides were significantly improved [12]. In other studies, muscle activation of the EO on both sides, the IO of the elevated side, and the gluteus maximus of the supporting limb was highly improved than those of the GBE [17]. Most studies have reported that the trunk muscles on the elevated side were more activated than the trunk muscles on the supporting side $[11,12,14,17]$.

As described above, various studies have confirmed that SLBE improves muscle activation in the abdominal area. However, most of the studies have focused on the effect of SLBE on normal adults and their effects in comparison to other trunk stabilizing exercises $[11,12,14,17]$. Studies on the effect of SLBE on stroke patients focused more on its effects on balance abilities rather than muscle activity $[15,16]$.

In this study, SLBE was performed by using the affected leg for support against the floor while the unaffected leg was unraised and placed on the ground comfortably. The SLBE was designed as such because previous studies have shown that abdominal muscle activation is greatly induced as the angle of the raised leg increases to decrease dependence levels in stroke patients [26]

Muscle activation of the EO and IO of the affected side increased significantly, and the muscle asymmetry of the EO and IO improved significantly.

The significant improvement in muscle activity of the EO and IO on the affected side after SLBE seems to be due to the strength generated in the abdominal area to compensate for changes in posture $[12,14,27,28]$. And it may be due to SLBE performed in this study used the affected side. The RA on the affected side did not show any significant change, which demonstrates that the effect of SLBE on the RA muscle is not as significant as that on the EO or IO muscles.

The asymmetry of the EO and IO was also significantly improved, which may be due to an increase in muscle activation on the affected side [13]. However, the decrease in muscle activation on the unaffected side was not statistically significant. The cause of these results remains unknown. This may be due to the fact that the exercise intervention in this study did not involve raising the unaffected leg, or due to insufficient sample size or inappropriately controlled exercise interventions and other variables. Further research is required to examine the reason behind muscle activation on the unaffected side, which did not decrease significantly.

Although there are limitations to comparing this study to previous studies due to differences in duration time and period of exercise intervention, the period 
since the onset of stroke and other general conditions of the patients, and the type of exercise intervention, the biggest difference is the participant pool of research and the type of exercise intervention used in this study. Previous studies focus on the effects of SLBE and other types of bridge exercises on muscle activation in normal adults; this study chose SLBE as the exercise intervention for stroke patients, as a result of taking into consideration their clinical characteristics to confirm changes in muscle activation and muscle asymmetry. The results of this study confirmed that SLBE as an exercise intervention was effective in stabilizing the abdominal muscles in stroke patients.

This study had several limitations. First, because the number of participants was small and the study was conducted in patients with subacute stroke, it is difficult to generalize the results of the study. Further research with a larger sample size and comparative studies with chronic stroke patients are needed to reinforce the implications of the results. Second, this was a preliminary study that examined the immediate effects of SLBE on the muscle. Therefore, this study was conducted within a relatively short time frame and thus calls for a study in which the effects of SLBE can be examined over a longer period of time. Third, this study had no control groups; therefore, comparison of results was not performed. Later studies can compare different results from various methods of SLBE to identify the optimal condition in which the effects on abdominal muscle activation in subacute stroke patients are maximized. Fourth, this study only measured the muscle activation of the RA, EO, and IO muscles. Further research is needed to examine the muscle activation of other muscles in relation to performing SLBE, and improvement in functional abilities such as balance or gait abilities could also be examined further.

In conclusion, SLBE is effective in activating the abdominal muscles of stroke patients on the affected side and is a helpful exercise intervention that activates the muscles to transform asymmetric abdominal muscles into symmetric patterns.

\section{References}

1. Feigin VL, Lawes CM, Bennett DA, Anderson CS.
Stroke epidemiology: a review of population-based studies of incidence, prevalence, and case-fatality in the late 20th century. Lancet Neurol. 2003;2:43-53.

2. Brunner IC, Skouen JS, Strand LI. Is modified constraint-induced movement therapy more effective than bimanual training in improving arm motor function in the subacute phase post stroke? A randomized controlled trial. Clin Rehabil. 2012;26:1078-86.

3. Cabanas-Valdés R, Cuchi GU, Bagur-Calafat C. Trunk training exercises approaches for improving trunk performance and functional sitting balance in patients with stroke: a systematic review. Neuro Rehabilitation. 2013;33:575-92.

4. Mayo NE, Wood-Dauphinee S, Ahmed S, Gordon C, Higgins J, Mcewen S, et al. Disablement following stroke. DisabilRehabil. 1999;21:258-68.

5. Verheyden G, Vereeck L, Truijen S, Troch M, Herregodts I, Lafosse C, et al. Trunk performance after stroke and the relationship with balance, gait and functional ability. Clin Rehabil. 2006;20:451-8.

6. Klein CS, Brooks D, Richardson D, McIlroy WE, Bayley MT. Voluntary activation failure contributes more to plantar flexor weakness than antagonist coactivation and muscle atrophy in chronic stroke survivors. J ApplPhysiol (1985). 2010;109:1337-46.

7. McGill SM, Grenier S, Kavcic N, Cholewicki J. Coordination of muscle activity to assure stability of the lumbar spine. J ElectromyogrKinesiol. 2003;13:353-9.

8. Arokoski JP, Valta T, Kankaanpää M, Airaksinen O. Activation of lumbar paraspinal and abdominal muscles during therapeutic exercises in chronic low back pain patients. Arch Phys Med Rehabil. 2004;85:823-32.

9. Marshall PW, Murphy BA. Core stability exercises on and off a Swiss ball. Arch Phys Med Rehabil. 2005;86:242-9.

10. Stevens VK, Bouche KG, Mahieu NN, Coorevits PL, Vanderstraeten GG, Danneels LA. Trunk muscle activity in healthy subjects during bridging stabilization exercises. BMC MusculoskeletDisord. 2006;7:75.

11. Kong YS, Cho YH, Park JW. Changes in the activities of the trunk muscles in different kinds of bridging exercises. J Phys Ther Sci. 2013;25:1609-12.

12. García-Vaquero MP, Moreside JM, Brontons-Gil E, Peco-González N, Vera-Garcia FJ. Trunk muscle ac- 
tivation during stabilization exercises with single and double leg support. J ElectromyogrKinesiol. 2012;22:398-406.

13. Hodges PW, Richardson CA. Relationship between limb movement speed and associated contraction of the trunk muscles. Ergonomics. 1997;40:1220-30.

14. Yoon JO, Kang MH, Kim JS, Oh JS. Effect of modified bridge exercise on trunk muscle activity in healthy adults: a cross sectional study. Braz J Phys Ther. 2018;22:161-7.

15. Chung EJ, Kim JH, Lee BH. The effects of core stabilization exercise on dynamic balance and gait function in stroke patients. J Phys Ther Sci. 2013;25:803-6.

16. Song GB, Heo JY. The effect of modified bridge exercise on balance ability of stroke patients. J Phys Ther Sci. 2015;27:3807-10.

17. Cho MK, Bak JW, Chung YJ. The effects of performing a one-legged bridge with use of a sling on trunk and gluteal muscle activation. Phys TherRehabil Sci. 2016;5:70-7.

18. Jang EM, Kim MH, Oh JS. Effects of a bridging exercise with hip adduction on the EMG activities of the abdominal and hip extensor muscles in females. J Phys Ther Sci. 2013;25:1147-9.

19. Pereira LM, Marcucci FC, de Oliveira Menacho M, Garanhani MR, Lavado EL, Cardoso JR. Electromyographic activity of selected trunk muscles in subjects with and without hemiparesis during therapeutic exercise. J ElectromyogrKinesiol. 2011;21:327-32.

20. Stevens VK, Coorevits PL, Bouche KG, Mahieu NN, Vanderstraeten GG, Danneels LA. The influence of specific training on trunk muscle recruitment patterns in healthy subjects during stabilization exercises. Man Ther. 2007;12:271-9.

21. Shumway-Cook A, Woollacott M. Motor control: translation research into clinical practice. 3rd ed. Baltimore: Lippincott Williams \& Wilkins; 2001.

22. Feldwieser FM, Sheeran L, Meana-Esteban A, Sparkes V. Electromyographic analysis of trunk-muscle activity during stable, unstable and unilateral bridging exercises in healthy individuals. Eur Spine J. 2012;21 Supp12:S171-86.

23. Ryerson S, Byl NN, Brown DA, Wong RA, Hidler JM. Altered trunk position sense and its relation to balance functions in people post-stroke. J Neurol
Phys Ther. 2008;32:14-20.

24. Sackley CM. Falls, sway, and symmetry of weight-bearing after stroke. Int Disabil Stud. 1991;13:1-4

25. Hsieh CL, Sheu CF, Hsueh IP, Wang CH. Trunk control as an early predictor of comprehensive activities of daily living function in stroke patients. Stroke. 2002;33:2626-30.

26. Lee HJ, Shin KH, Byun SM, Jeong HS, Hong JS, Jeong SJ, et al. The changes of rectus abdominis muscle thickness according to the angle during active straight leg raise. Phys TherRehabil Sci. 2013;2:44-8

27. Cholewicki J, VanVliet JJ 4th. Relative contribution of trunk muscles to the stability of the lumbar spine during isometric exertions. Clin Biomech (Bristol, Avon). 2002;17:99-105.

28. Aruin AS, Latash ML. Directional specificity of postural muscles in feed-forward postural reactions during fast voluntary arm movements. Exp Brain Res. 1995; 103:323-32. 